\title{
Mass Transfer in Binary Systems: A Numerical Approach
}

\author{
C.-P. Lajoie and A. Sills \\ Department of Physics \& Astronomy, McMaster University, Hamilton, ON L8S 4M1, Canada \\ email: lajoiec@mcmaster.ca, asills@mcmaster.ca
}

\begin{abstract}
We present preliminary work on the formation scenario of blue straggler stars by mass transfer in binary systems. More precisely, using Smoothed Particle Hydrodynamics (SPH), we want to model only the outer parts of the stars in order to get a much greater spatial resolution of the mass transfer flow itself. The inner boundary conditions are achieved using the so-called ghost particles and by replacing the inner mass by a central point mass. Stability of this central point mass is crucial, and it is shown that we get reasonable results. These simulations should give us indications on which layers of the donor star are actually transferred to the other star as well as how mass is transferred and how it settles on the accretor. This work is aimed at getting distinct observational signatures which would help identifying the dominant formation mechanism of blue straggler stars.
\end{abstract}

Keywords. binaries: close, blue stragglers, methods: numerical

\section{Introduction}

Globular clusters are dense stellar systems in which dynamical interactions between stars can significantly alter the stellar populations. It is thus important to understand how these interactions occur and what they result in if we want to better constrain the dynamical and formation histories of globular clusters. For example, blue stragglers, which are found above the main-sequence turn-off point in color-magnitude diagrams, are thought to be created either by collisions between two main-sequence stars or by mass transfer in primordial binaries. Observationally, however, it is rather difficult to distinguish between these two scenarios (see, e.g., Ferraro et al. 2006), so a better theoretical understanding of how these objects form may help identifying the formation of blue stragglers.

\section{Numerical Approach}

We address this problem by using our Smoothed Particle Hydrodynamics (SPH) code, which is based on the early version of Benz (1990) and, more recently, Bate, Bonnell \& Price (1995). Briefly, this methods treats fluids elements as smoothed particles, which are subject to hydrodynamical forces such as pressure gradient and viscosity (Balsara 1995), as well as gravity (see Benz et al. 1990). Particles are smoothed over a volume defined by their smoothing length, which in turn is determined by the requirement that each particle must have a given number of neighbors. Thus, the total number of particles puts limits on the spatial resolution of the simulation.

To get a detailed description of the mass transfer flow between the two stars as well as how the matter settles onto the accretor, we need high spatial resolution. In order to reach such a high resolution without using extremely large number of particles, we intend to model only the outer parts of the stars, as shown in Fig. 1a. The boundary 

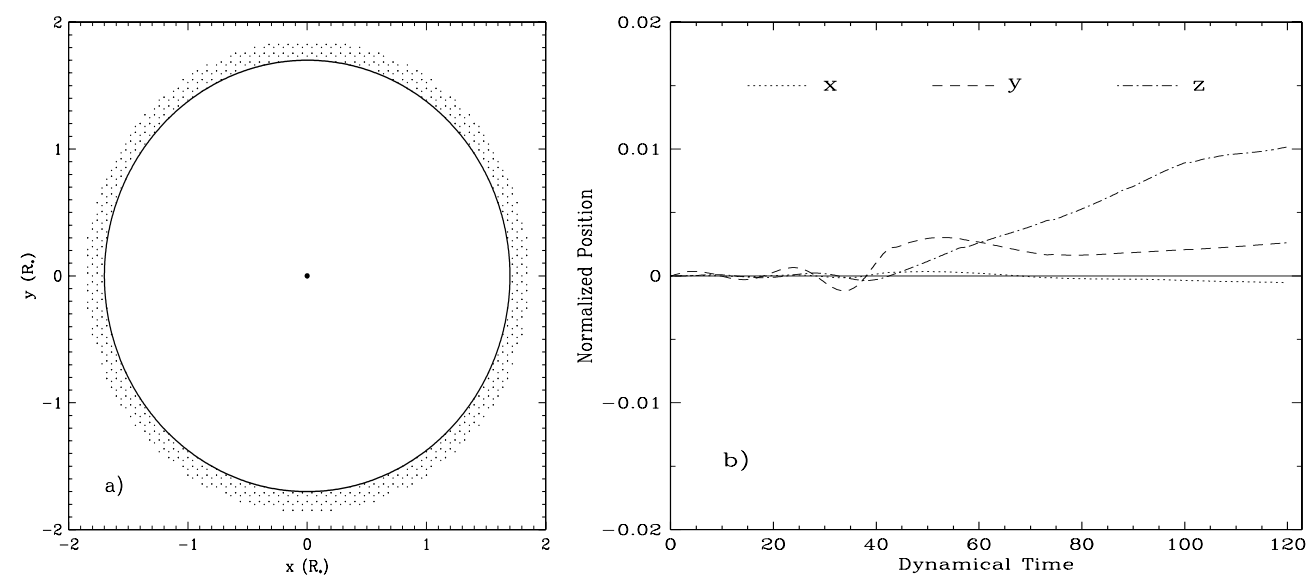

Figure 1. a) Initial configuration of our model star, with the SPH particles represented by small dots, the boundary shown as the solid line and the central point mass shown as the solid dot at the centre of the star. b) Normalized position of the central point mass as a function of time for the initial configuration shown in a). The solid line shows the equilibrium position of the point mass, which initially corresponds to the centre of the star.

conditions are achieved using the so-called ghost particles (see, e.g., Morris, Fox \& Zhu 1997) and the SPH particles located inside this boundary are replaced by a point mass with the same total mass. We have tested the stability of the central point mass for an isolated star, and Fig. 1b shows that it drifts only slightly (i.e. one percent of the star's initial radius) over 120 dynamical times.

\section{Future Work}

The simultaneous implementation of boundaries for two stars is now underway, and following the encouraging results of $\S 2$, we should start running simulations of mainsequence binaries in the near future. We think these simulations will be useful in identifying distinct observational signatures of blue stragglers formed by mass transfer and help identifying their main formation scenario.

\section{Acknowledgements}

This work was supported by NSERC Canada and made possible by the facilities of the Shared Hierarchical Academic Research Computing Network (SHARCNET: www.sharcnet.ca).

\section{References}

Balsara, D. S. 1995 Jour. Comput. Physics 121, 357

Bate, M. R., Bonnell, I. A., \& Price, N. M. 1995, MNRAS 277, 362

Benz, W. 1990, in: Buchler J. R. (ed.), The Numerical Modeling of Nonlinear Stellar Pulsations: Problems and Prospects, Kluwer, Dordrecht, p. 269

Benz, W., Bowers, R. L., Cameron, A. G. W., \& Press, W. 1990 ApJ 348, 647

Ferraro, F. R., Sabbi, E., Gratton, R., Piotto, G., Lanzoni, B., Carretta, E., Rood, R. T., Sills, A., Fusi Pecci, F., Moehler, S., Beccari, G., Lucatello, S., \& Compagni, N. 2006, ApJ 647, L53

Morris, J. P., Fox, P. J., \& Zhu, Y. 1997 Jour. Comput. Physics 136, 214 